
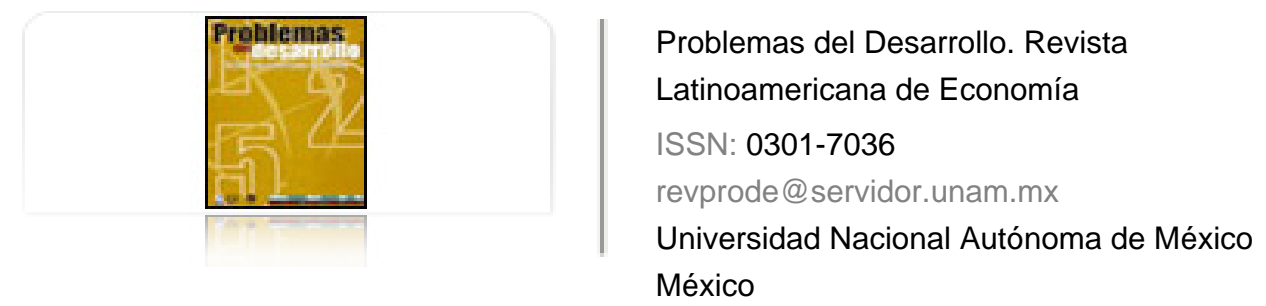

Dabat, Alejandro; Ordóñez, Sergio; Rivera Ríos, Miguel A.

La reestructuración del cluster electrónico de Guadalajara (México) y el nuevo aprendizaje tecnológico Problemas del Desarrollo. Revista Latinoamericana de Economía, vol. 36, núm. 143, octubre-

diciembre, 2005, pp. 89-111

Universidad Nacional Autónoma de México

Distrito Federal, México

Disponible en: http://www.redalyc.org/articulo.oa?id=11820081005

Cómo citar el artículo

Número completo

- Más información del artículo

Página de la revista en redalyc.org

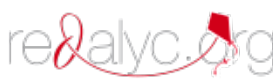

Sistema de Información Científica

Red de Revistas Científicas de América Latina, el Caribe, España y Portugal Proyecto académico sin fines de lucro, desarrollado bajo la iniciativa de acceso abierto 


\title{
LA REESTRUCTURACIÓN DEL CLUSTER ELECTRÓNICO DE Guadalajara (MÉXICO) Y El nUeVo APRENDIZAJe TECNOLÓGICO
}

\author{
Alejandro Dabat* \\ Sergio Ordóñez ${ }^{* *}$ \\ Miguel A. Rivera Ríos***
}

Fecha de recepción: 20 de mayo de 2005. Fecha de aceptación: 10 de octubre de 2005.

\section{Resumen}

El cluster electrónico de Guadalajara se reestructuró como consecuencia de los severos efectos de la crisis global de las telecomunicaciones de 2001-2002. Aunque la caída de las exportaciones de productos electrónicos se había superado en 2004, dicha crisis implicó un desplazamiento de producción hacia China, que paralelamente consolidó su papel como potencia mundial en electrónica. La localidad en México tendió a adaptarse a las nuevas condiciones de mercado, especializándose en productos de mayor valor agregado generados en series menores. Un aspecto sobresaliente de ese cambio de perfil productivo en el cluster ha sido la emergencia de un nuevo tipo de empresa doméstico-local que provee servicios avanzados a las empresas líderes conocidas como original equipment manufacturing (OEM). El artículo, basado en trabajo de campo, se centra en el estudio del nuevo aprendizaje tecnológico entre esas empresas locales.

Palabras clave: industria electrónica en México, aprendizaje tecnológico, cluster y desarrollo industrial.

\footnotetext{
* Investigador titular del CRIM, comisionado en el Insituto de Investigaciones Económicas (IIEC UNAM). Correo electrónico: dabat@servidor.unam.mx

** Investigador titular A de tiempo completo (interino) IIEC-UNAM. Correo electrónico: serorqu@avantel.net

*** Investigador titular del posgrado de la Facultad de Economía (FE UNAM).
} 


\begin{abstract}
The electronics cluster in Guadalajara has been re-structured due to the severe effects of the global telecommunications crisis of 2001-2002. Although the drop in exports of electronic products had been overcome by 2004, this crisis implied a displacement of production to China, which, at the same time, consolidated that country's role as a world power in electronics. The locality in Mexico has tended to adapt itself to the new market conditions, specializing in products with higher aggregate value, generated in shorter series. One outstanding aspect of that change of production profile in the cluster has been the emergence of a new type of local domestic company that provides advanced services to leading companies known as original equipment manufacturing (OEM). This article, based on field-work, focuses on the study of the new technological learning process among these local companies.
\end{abstract}

Key words: electronics industry in Mexico, technological learning, cluster, industrial development.

\title{
Résumé
}

La restructuration du cluster électronique de Guadalajara a été la conséquence des effets sévères de la crise globale des télécommunications de 2001-2002. Bien que la chute des exportations de produits électroniques avait été surmontée en 2004, cette crise a impliqué un déplacement de production vers la Chine, qui a consolidé, parallèlement, son rôle de puissance mondiale en électronique. La tendance de la localité au Mexique a été de s'adapter aux nouvelles conditions du marché, en se spécialisant dans des produits d'une plus grande valeur ajoutée générés en plus petites séries. Un aspect remarquable de ce changement de profil productif dans le cluster a été l'émergence d'un nouveau type d'entreprise domestique locale qui pourvoit en services approfondis les entreprises leader connues sous le nom de original equipment manufacturing (OEM). L'article, qui se base sur un travail de champ, se centralise sur l'étude du nouvel apprentissage technologique entre ces entreprises locales.

Mots-cléfs: industrie électronique au Mexique, apprentissage technologique, cluster et développement industriel.

\section{Resumo}

O cluster eletrónico de Guadalajara foi reestruturado como consequência dos severos efeitos da crise global das telecomunicações de 2001-2002 Ainda que a queda das exportações de produtos eletrónicos tinha sido superada em 2004, tal crise implicou um deslocamento de produção à China, que ao mesmo tempo consolidou seu papel como potência mundial na eletrónica. A localidade no México tendeu a adatar-se às novas condições do mercado se especializando em produtos de maior valor agregado gerados em séries menores. Um aspecto relevante dessa mudança de perfil produtivo no cluster foi a emergência dum novo tipo de empresa doméstico-local que oferece serviços avançados às empresas líderes conhecidas como original equipment manufacturing (OEM). O artigo, baseado no trabalho de campo, centra-se no estudo da nova aprendizagem tecnológica entre estas empresas locais.

Palavras chave: indústria eletrónica no México, aprendizagem tecnológica, cluster e desenvolvimento industrial.

\section{DeSarrollo}




\section{Introducción}

$\mathrm{E}$

1 cluster electrónico de Guadalajara, en el Occidente de México, representa el ejem-

plo más avanzado de desarrollo territorial derivado de la reinserción internacional

mexicana, iniciada en los años ochenta, así como de la integración a la economía de Norteamérica, emblematizada por el TLCAN. Habiendo constituido uno de los pilares del auge exportador mexicano de los noventa, atrajo la atención de investigadores nacionales y extranjeros, quienes centraron sus estudios en las transformaciones verificadas en dicha década. Pero la crisis mundial del sector electrónico informático (SE-I) ${ }^{1}$ de 2001-2002 implicó un fuerte impacto en la industria electrónica de exportación de México, en particular en el cluster de Guadalajara, sin que ese proceso, por ahora, haya llamado la atención de los investigadores, como sucedió con el auge exportador. Para analizar los aspectos más sobresalientes de la crisis y de la reestructuración de las actividades de esa localidad (con su expresión de lo que llamaremos nuevo aprendizaje tecnológico entre empresas locales) se tomó como base algunos de los estudios más destacados arriba mencionados y los resultados de un trabajo de campo efectuado en el cluster de Guadalajara. ${ }^{2}$

La hipótesis adoptada, sometida además a verificación en la actividad de campo, es que con la reestructuración del cluster se dio lugar a un primer e incipiente pasaje, al cual llamamos segunda modalidad de aprendizaje tecnológico, ${ }^{3}$ entre empresas domésticolocales integradas a redes de proveedores, centralizadas por empresas original equipment

1 Denominamos sector electrónico-informático (SE-I) a lo que habitualmente se conoce como sector de tecnologías de la información y las comunicaciones (STIC), por considerar que SE-I es una designación más correcta, al referirse a los sectores productivos que lo componen más que a las tecnologías en las cuales se basa (Dabat y Ordóñez, en prensa).

2 La investigación de campo acerca de la industria electrónica comenzó en 2001, con la conducción de Sergio Ordóñez, centrada principalmente en el cluster de Guadalajara. Esa etapa culminó con una serie de entrevistas efectuadas entre el 21 y el 25 de noviembre de 2004 a directores de empresas, personal del gobierno estatal (Secretaría de Economía), de la Cámara de la Industria Electrónica (CANIETI), de la Cadena Productiva de la Electrónica (CADELEC) y del Centro de Investigación y Estudios Avanzados del Instituto Politécnico Nacional (CINVESTAV). En la tercera parte del artículo se presenta la relación de personas entrevistadas. Esta etapa de la actividad de campo se efectúo con el apoyo de CONACYT, dentro del proyecto "La nueva economía del conocimiento y el aprendizaje en México. Un enfoque comparativo y sectorial”, coordinado por Miguel A. Rivera Ríos, con la participación de Alejandro Dabat y Sergio Ordóñez, quienes en equipo llevaron a cabo la actividad final de campo.

3 Utilizamos aprendizaje tecnológico en un sentido equivalente al de escalamiento industrial (upgrading) de Gereffi $(1995,2004)$, en tanto ambos apuntan a la idea de acumulación de capa cidades tecnológicas y organizativas requeridas para avanzar en la escala de desarrollo y, por ende, en la cadena de valor mundial. A su vez, el fundamento del aprendizaje tecnológico es la instauración de lo que llamamos, siguiendo a Bell y Albu (1999), sistemas de conocimiento en la empresa.

\section{DeSarrollo}


manufacturing (OEM), poseedoras de patentes y centradas en diseño (lo que le da la connotación original design manufacturing, ODM). ${ }^{4}$ La primera modalidad de aprendizaje es la que se ha puesto de manifiesto en la literatura de clusters en México y consiste en la inserción de la empresa local en la parte baja de la cadena de proveedores, ofreciendo productos de bajo valor agregado que se suelen designar como necesarios; se llegó a abrigar la expectativa de que partiendo de un nivel bajo, a la larga habría un ascenso o upgrading en el sentido propuesto por Gereffi (1995 y 2004). En la investigación de campo se comprobó que para el caso de Guadalajara, este medio de ascenso estaba virtualmente cerrado y que, inclusive, el acceso de empresas locales a la cadena de proveedores de esos productos se había vuelto muy restrictivo desde principios de los noventa. Las razones, como veremos, remiten a cambios organizativos en la industria electrónica global, acelerados por la intensificación de la competencia mundial que desemboca en la proliferación de contratistas globales de manufactura o contract manufacturers (CM). ${ }^{5}$

No tenemos elementos para sostener que ese primer pasaje a una segunda modalidad de aprendizaje opere más allá del cluster de Guadalajara, es decir, que se haya propagado a otras aglomeraciones del norte de México, las cuales son asiento de operaciones de empresas internacionales como en Baja California o Chihuahua. Hay participación importante de empresas locales en la industria de autopartes, pero se requerían investigaciones más amplias y focalizadas en el aprendizaje local para verificar la hipótesis anterior en un radio territorial más grande. También la investigación de campo centrada en Guadalajara

4 En la literatura de cadenas productivas y redes empresariales, los conceptos de original equipment manufacturers (OEM) y original design manufacturers (ODM) se han utilizado en dos sentidos. Por un lado, autores como Gereffi $(1995,2004)$ y Hobday (1995) lo aplican a las empresas subcontratis tas asiáticas que manufacturan y diseñan para empresas trasnacionales de marca, mientras que estas últimas, en lugar de OEM ODM, serían más bien original brand manufacturers (ОВM). A di ferencia de esta conceptuación, los estudiosos del cluster de Guadalajara llaman empresas OEM ODM a la transnacional de marca en pleno proceso de desprendimiento de las actividades manufactureras, concentrada en I\&D, diseño y mercadeo. Para conservar la terminología adoptada en México, la cual hemos utilizado en otros trabajos, continuaremos llamando OEM ODM a la trasnacional líder.

5 La estructura modular de las empresas internacionales, dentro de la cual se inscriben los contract manufacturers, comenzó a propagarse desde fines de los ochenta y principios de los noventa. La empresa que le dio prominencia en los medios de difusión a esa estructura modular fue Apple Computer, cuando en 1996 vendió sus plantas de manufactura en Estados Unidos a SCI, quien se comprometió a seguir fabricando las computadoras sobre la base del diseño proporcionado por su nuevo cliente, lo cual puede considerarse el modelo de la relación entre las OEM y CM (Sturgen, 2002). Para fines de los noventa las firmas electrónicas más importantes - como IBM, Nortel, $3 \mathrm{Com}$, HP y Lucent, entre otras-, no sólo compraban a proveedores externos, sino que habían ven dido sus plantas domésticas y en el extranjero. También las compañías más jóvenes - como Sun Microsystems, Cisco System, Silicon Graphics-, ya habían adoptado esa misma organización (Sturgen, op. cit.). Los mayores contratistas manufactureros del mundo operan en México y cuatro de las cinco primeras (Solectron, Flextronics International, Sanmina/sci y Jabil Circuit) en Guadalajara desde el arranque mismo del boom exportador. Desde comienzos de la nueva década, las CMS comenzaron a llamarse electronic manufacturers services (EMS).

\section{DeSarrollo}


y municipios colindantes debe tener continuidad para verificar si ese primer brote perdura y se amplifica.

Para alcanzar los objetivos de investigación presentados en la hipótesis, se dividió la exposición en tres partes. La primera, para fines comparativos, revisa la etapa previa comprendida entre mediados de los ochenta y la culminación del auge exportador hacia 2001, recurriendo a parte de la amplia literatura e investigación existentes acerca de la misma. Dentro de ese tratamiento se incluye una propuesta de conceptuación de cluster formulada a partir de comparaciones internacionales y se plantea un escueto marco teórico para apoyar el estudio de las modalidades de aprendizaje tecnológico empresarial. En el segundo apartado se brinda el marco de análisis de la crisis y reestructuración del cluster, tomando como ángulo la evolución reciente de la industria electrónica mundial; en este apartado se hace hincapié en las repercusiones de la competencia china, el principal productor mundial de equipo electrónico, apoyándose en las repercusiones de la primera crisis del SE-I. En la tercera parte se estudia, con base en la investigación de campo, el aprendizaje de segunda modalidad entre las empresas locales, pero sobre todo, se trata de definir cuál es la base institucional de este ascenso industrial que ha sido muy elusivo no sólo en México, sino en el conjunto de los países de América Latina. No se pretende sobredimensionar la importancia de este ascenso, que es a todas luces embrionario, pero se subraya que la experiencia de México reproduce un proceso dictado por los requerimientos de las empresas globales que demandan trabajo técnico de alto nivel y de ingeniería en escala considerable y que tiende a ser satisfecha por países de desarrollo medio que cuentan con una nueva ventaja competitiva: cuadros calificados a salarios que son una fracción de la existente en países desarrollados.

\section{Estructura y modalidades de desarrollo del cluster hasta el fin del auge}

El origen, etapas de desarrollo y primer tipo de aprendizaje

En tanto prevalecía en el nivel nacional una modalidad de industrialización basada en la protección del mercado interno, la intervención estatal directa y el liderazgo formal de la empresa nacional (Peres, 1990; Rivera, 2000) se inició la primera etapa de desarrollo del cluster al establecerse en Guadalajara, a fines de los años sesenta. Dicha etapa comenzó con una modesta industria eléctrica, y en menor medida electrónica, cuyos pilares iniciales fueron las filiales de Motorola y Burroughs, las cuales, al ampliarse en 1972 la cobertura territorial del Programa para la Industrialización de la Franja Fronteriza Norte o Programa de Maquila, comenzaron a importar partes y componentes libres de aranceles para ensamblarlos y reexportarlos, lo que dio origen a lo que se ha conocido como industria 
maquiladora de exportación de productos eléctrico-electrónicos en la localidad (Palacios, 2003:235). ${ }^{6}$

A principios de los ochenta, con la normatividad del Programa para la Promoción de Equipo de Cómputo, Modulares y Periféricos dado a conocer en 1981 (conocido como Plan Calcul versión original) se empezaron a definir, de manera aún difusa, nuevas perspectivas de lo que hasta entonces era puramente una industria de ensamble simple o maquiladora de exportación. El referido programa, pretendiendo revitalizar la sustitución de importaciones y complementarla con la exportación (dando prioridad a la participación del capital nacional, la integración nacional de la industria y el desarrollo de una tecnología propia), ${ }^{7}$ propicia el surgimiento de varias empresas de capital mexicano productoras de computadoras de marca propia, como Compumex, Microton y Wind Computers. ${ }^{8}$ Éstas posteriormente desaparecerían o renunciarían a producir con marca propia, abocándose a la elaboración de componentes o a proporcionar servicios relacionados, como resultado del encarecimiento de las partes importadas derivado de la devaluación de 1982 y la fuerte depreciación del tipo de cambio durante 1986 y 1987 (Palacios, 2001; Dabat y Ordóñez, op. cit.), lo cual canceló esta modalidad de desarrollo del cluster hacia mediados de los ochenta.

En 1985 dio inicio la segunda etapa de desarrollo, cuando las prioridades del Plan Calcul se modificaron en favor del desarrollo de la productividad y la competitividad industriales, ${ }^{9}$ con el propósito de autorizar a IBM para que construyera una nueva planta con $100 \%$ de capital extranjero en el municipio de El Salto. Esta decisión es parte de un proceso de transición del ensamble de máquinas de escribir electrónicas al de computadoras y el subensamble de componentes de circuitos electrónicos (Ordóñez, 2004). ${ }^{10}$

6 Los principales productos fueron radios, cables y fuentes de poder, entre otros. En los inicios, el ensamble de equipo de telecomunicaciones y computadoras tenía una participación muy modesta (Warman, 1994).

7 Las empresas inscritas en el plan debían contar con 51\% de capital mexicano y gozarían de estímulos fiscales, en la forma de créditos de impuestos, así como de protección comercial a la importación de productos terminados, material periférico, piezas de recambio y componentes. Por otro lado, podrían importar partes y componentes a tasas preferenciales (Peres, op. cit.).

8 El surgimiento de estas empresas productoras de marcas propias es precedido por la empresa Electrónica Zonda fundada en 1970 y que, hacia finales de los años sesenta, derivó en cuatro empresas hijas: Sonimex (equipo de audio), Refrimex (refrigeradores), Tvmex (televisores avanzados) y Compumex (computadoras personales). Véase Palacios (2001).

9 Con esta modificación, las empresas podían seguir el medio establecido por la versión original del plan o, bien, aquella marcada por la versión modificada, en cuyo caso debían contar con alto coefi ciente de exportación/importación: 3/1 (en caso de una participación de capital nacional mayoritaria, el coeficiente descendía a 1:1) (Peres, ibid.). En opinión de Peres, la aceptación del proyecto de IBM representó la derrota del grupo gubernamental que respaldaba una política industrial tradicional y fortaleció, en cambio, al grupo "aperturista" (ibid::90). En realidad, el grupo de la Secretaría de Industria y Comercio que respaldaba el programa de 1981 y su reformulación posterior no se identificaba con una política industrial tradicional, como la que prevaleció en los años sesenta setenta, sino otra de transición, ajustada formalmente a las propuestas de Balassa (1983).

10 Entre las razones que determinan que las empresas extranjeras decidan localizarse en Guadalajara se encuentran: a) la existencia de fuerza de trabajo a bajo costo, calificada, de universidades, centros 
LA REESTRUCTURACIÓN DEL CLUSTER ELECTRÓNICO DE GUADALAJARA (MÉXICO)

Cuadro 1

Sistemas y capacidades de conocimiento en cuatro empresas locales tradicionales de Guadalajara (mediados de los años noventa)

\begin{tabular}{lcccc}
\hline & $\begin{array}{c}\text { Desarrollo de } \\
\text { nuevos procesos }\end{array}$ & $\begin{array}{c}\text { Desarrollo de } \\
\text { nuevos productos }\end{array}$ & $\begin{array}{c}\text { Avances de gestión y } \\
\text { control de calidad }\end{array}$ & $\begin{array}{c}\text { Escalamiento } \\
\text { en productos }\end{array}$ \\
\hline Yamaver & $\mathrm{x}$ & $\mathrm{x}$ & $\mathrm{x}$ & \\
Ureblok & $\mathrm{x}$ & $\mathrm{x}$ & $\mathrm{x}$ & $\mathrm{x}$ \\
Compuworld & $\mathrm{x}$ & $\mathrm{x}$ & $\mathrm{x}$ & $\mathrm{x}$ \\
E. Pantera & $\mathrm{x}$ & $\mathrm{x}$ & $\mathrm{x}$ & $\mathrm{x}$ \\
\hline
\end{tabular}

Fuente: elaborado conforme a información proveniente de Dussel (1998:52-59).

La necesidad de cumplir con el coeficiente de exportación/importación impuesto por el Plan Calcul llevó a IBM a poner en práctica dos iniciativas que enmarcaron la primera modalidad de aprendizaje tecnológico para las empresas locales: $a$ ) la fundación del Centro de Tecnología de Semiconductores (CTS), y b) un programa de formación de proveedores. La creación del cTs en 1988 fue resultado de un acuerdo auspiciado por la Secretaría de Fomento Industrial, para que IBM se asociara con el CINVESTAV-IPN, con el objetivo de crear un laboratorio destinado al desarrollo de capacidades tecnológicas endógenas en diseño de semiconductores que sirviera de soporte al aprendizaje tecnológico local. De forma complementaria, IBM puso en práctica un programa de proveedores que fue el punto de partida para la integración de empresas locales y después fue adoptado por otras empresas extranjeras como Hewlett-Packard.

El impacto en el aprendizaje tecnológico local quedó limitado a un pequeño grupo de empresas que se especializaron en productos de categoría inferior, proceso que, sin embargo, constituyó el germen de la formación de lo que Bell y Albu (op. cit.) denominaron sistemas de conocimiento (véase Cuadro 1$).{ }^{11}$

La asistencia técnica y gerencial que recibieron de las corporaciones extranjeras fueron determinantes para obtener el aprendizaje tecnológico necesario a fin de establecer los sistemas de conocimiento para nuevos productos y procesos. Este avance exigió a dichas

tecnológicos y educativos con énfasis en preparación técnica e ingeniería; $b$ ) una cultura artesanal importante para ese tipo de trabajo y el ensamble de productos; c) proximidad geográfica con Esta dos Unidos y disponibilidad de infraestructura de transportes (aeropuerto y puertos internacio nales), y d) calidad de vida aceptable para el ciudadano estadounidense. Todos esos atributos de la ciudad quedaron de manifiesto en entrevistas realizadas en 1999, en ocho empresas electrónicas ubicadas en los alrededores y reconocidas por los especialistas en el tema.

11 Los flujos de conocimiento entre empresas, al interior o entre agentes empresariales y no empresariales, se distinguen de los flujos de materiales, aunque frecuentemente se traslapen con ellos. Ese conocimiento permite instituir el cambio tecnológico en la empresa, en tanto estén creadas las capacidades apropiadas para ello. Éstas tienen diferentes grados de complejidad, que van desde las rutinas requeridas para garantizar la eficiencia de los sistemas de producción, hasta las requeridas para diseñar nuevos productos, nueva maquinaria y renovar los procesos y canales de abastecimiento y distribución (Bell y Albu, ibid.:1723).

\section{DeSarrollo}


empresas sustanciales inversiones en equipo, instalaciones y capacitación de obreros especializados, técnicos e ingenieros. La principal limitante en la instauración de los sistemas de conocimiento fue la alta dependencia de la importación de insumos que en algunos casos llegó a 90\% del abastecimiento (Dussel, op. cit.:52), pues los sustitutos nacionales, cuando los había, eran de inferior calidad (ibid.).

La tercera etapa del desarrollo del cluster tiene lugar a partir de la primera mitad de los años noventa, cuando el TLCAN sustituyó al Plan Calcul como instrumento de regulación de la industria, lo cual abrió el camino para que las empresas oEM promovieran la radicación en la localidad de sus contratistas manufactureros internacionales (CM), con el consecuente incremento de la inversión extranjera en el cluster, hasta constituir $47 \%$ en la industria electrónica en su conjunto entre 1996 y 1999 (un total acumulado de 1618 millones de dólares (CADELEC, 2004). Lo anterior cancela prácticamente la primera modalidad de aprendizaje tecnológico e incorporación desde abajo en la cadena de valor de las empresas locales, pues los nuevos cm se instalan en la localidad atrayendo simultáneamente a sus propios proveedores internacionales de partes y componentes fundamentales, ${ }^{12}$ con la consiguiente formación de nuevas cadenas e incorporación simultánea en cadenas globales, de las cuales quedan excluidas, en lo fundamental, las empresas locales (Dussel, 1998; Ordóñez, 2004 y 2005).

Es con esta modalidad de desarrollo que tiene lugar el auge exportador del cluster durante los años noventa, lo cual se traduce en un incremento vertiginoso de su participación en las exportaciones totales de la industria electrónica de $15.5 \%$ en 1994 a más de $31 \%$ en 1999, año en el cual alcanza su máximo, paralelamente al aumento del peso de las exportaciones de la industria electrónica en el total manufacturero de 17\% en 1994 al nivel máximo de $24 \%$ en 2001, así como de la participación de Estados Unidos como destino principal de las exportaciones, como lo muestra el Cuadro 2.

El cluster de Guadalajara se especializó en esta etapa en equipo de cómputo, de telecomunicaciones y partes y componentes producidos en grandes series, que requieren de una variedad reducida de componentes, pero el patrón de especialización se vio afectado por la crisis de 2001 y 2002 como se verá más adelante.

Agentes participantes en la etapa madura

de desarrollo durante el auge

La organización industrial que se consolidó en los noventa estuvo definida por la participación de los siguientes agentes empresariales:

12 Dussel (2003) insiste correctamente en que la generalización de los diferentes programas de importación temporal (PITEX, ALTEX y de maquiladoras) dejó adicionalmente en desventaja a los proveedores locales, ya que premiaba fiscalmente la importación de partes y componentes.

\section{DeSarrollo}


Cuadro 2

Exportaciones de la industria electrónico-informática

Total nacional sectorial y estatal, 1990-2003

(millones de dólares)

\begin{tabular}{|c|c|c|c|c|c|c|c|}
\hline \multirow[b]{2}{*}{ Año } & \multirow{2}{*}{$\begin{array}{c}1 \\
\text { Exportaciones } \\
\text { totales } \\
\text { nacionales }\end{array}$} & \multicolumn{2}{|l|}{2} & \multicolumn{2}{|c|}{$\begin{array}{c}3 \\
\text { Exportaciones }\end{array}$} & \multicolumn{2}{|c|}{$\begin{array}{c}4 \\
\text { Exportaciones }\end{array}$} \\
\hline & & $\begin{array}{l}\text { Exportaciones } \\
\text { electrónicas }\end{array}$ & $\begin{array}{l}2 / 1 \\
\%\end{array}$ & $\begin{array}{c}\text { electrónica a } \\
\text { Estados Unidos }\end{array}$ & $\begin{array}{l}3 / 2 \\
\%\end{array}$ & $\begin{array}{c}\text { electrónica a } \\
\text { Jalisco }\end{array}$ & $\begin{array}{c}4 / 3 \\
\%\end{array}$ \\
\hline 1990 & 26838 & 748 & 2.8 & 416 & 55.6 & - & - \\
\hline 1991 & 42687 & 1009 & 2.4 & 639 & 63.3 & - & - \\
\hline 1992 & 46196 & 6419 & 13.9 & 3427 & 53.4 & - & - \\
\hline 1993 & 51886 & 7554 & 14.6 & 4163 & 55.1 & - & - \\
\hline 1994 & 60882 & 10347 & 17.0 & 5057 & 48.9 & 1600 & 15.5 \\
\hline 1995 & 79541 & 12563 & 15.8 & 8024 & 63.4 & 2300 & 18.3 \\
\hline 1996 & 96000 & 15739 & 16.4 & 10211 & 64.9 & 3500 & 22.2 \\
\hline 1997 & 110431 & 19764 & 19.7 & 13666 & 69.1 & 5200 & 26.3 \\
\hline 1998 & 117459 & 23949 & 20.4 & 18362 & 76.7 & 6400 & 26.7 \\
\hline 1999 & 136391 & 29396 & 21.6 & 23296 & 79.2 & 9144 & 31.1 \\
\hline 2000 & 166455 & 37577 & 22.6 & 31017 & 82.5 & 9860 & 26.2 \\
\hline 2001 & 158443 & 38466 & 24.3 & 31091 & 80.8 & 10529 & 27.4 \\
\hline 2002 & 160762 & 37721 & 23.5 & 29453 & 78.1 & 9976 & 26.4 \\
\hline 2003 & 164922 & 36215 & 22.0 & 28877 & 79.4 & 7872 & 21.7 \\
\hline 2004 & 202735 & 38751 & 19.1 & 32181 & 83.0 & 8500 & 21.9 \\
\hline
\end{tabular}

Notas: a) La información de la diferentes columnas no es totalmente compatible por significativas diferencias entre las distintas fuentes utilizadas, especialmente entre las mexicanas y estadounidenses. $b$ ) Las exportaciones electrónicas totales han sido tomadas sobre la base del sistema clasificatorio y las cifras de Naciones Unidas, códigos 751, 752, 753, 641, 642, 643, 644, 774, 776, 872, 873 y 874 de la Clasificación Uniforme del Comercio Internacional (CUCI).

Fuentes: INEGI: www.inegi.gob.mx, Banco de Información Económica; onu, International Trade of Statistics Yearbook 1990-1998, INTRACEN, 1999-2003, http://www.intracen.org; De 2000-2004 us Bureau of Census: http://censtats.census.gov/sitc/sitc.shtml; de 1990 a 1999 datos ajustados de la Secretaría de Economía, en www.economia.gob.mx; CADELEC, en www.cadelec.com.mx.

1) las filiales de varias de la principales empresas transnacionales del sector actuando como OEM-ODM, de propiedad 100\% extranjera (IBM, HP, NEC, Motorola, Lucent, Siemens, entre otras) que poseen patentes y marcas, diseñan productos y en un inicio centralizan las redes de proveedores, tendiendo a delegar la gestión de la cadena de valor en los contratistas manufactureros.

2) el grupo contratistas manufactureros o cM, igualmente extranjeros (Solectron, Flextronics International, Sanmina/sci y Jabil Circuit), los cuales manufacturan por contrato conforme pedido y diseño de las oEM. Su fuerza deriva (a diferencia de contratistas especializados) de la capacidad en manufactura genérica, operando con base en enormes economías de escala y el rango global de sus operaciones.

3) los proveedores internacionales de partes y componentes que abastecen a los contratistas manufactureros in situ, ya sea en parques industriales de la localidad o en instalaciones independientes.

\section{Desaarrollo}


4) un número muy pequeño de empresas de gestación interna, del tipo desprendimiento ( pin offs) o fundación (start ups). Se trata de firmas que se gestan en el contexto de la iniciativa empresarial local y representan el elemento empresarial endógeno en el cual se centraliza el aprendizaje tecnológico de la firma mexicana.

Esos agentes se relacionan en redes de proveedores, que se pueden estudiar como una sucesión de círculos en los cuales se organizan las redes de proveedores; de acuerdo con una investigación pionera de Dussel, son tres. El primer círculo o epicentro está constituido por empresas OEM (op. cit.:41), las cuales ocupan la posición líder, en tanto son las coordinadoras de encadenamientos globales dirigidos por el productor, acorde con la terminología de Gereffi (1995). El segundo está representado principalmente por los contratistas manufactureros globales (Palacios, op. cit.; Dussel, op. cit.) que, como vimos, tienen capacidad de organización productiva, logística y coordinación cualitativamente superiores. En el tercer círculo participan los que Dussel llama proveedores de productos "necesarios", es decir, complementos como empaques, inyecciones de plástico, flejes, cables. Como se discutió anteriormente, en la segunda etapa del desarrollo del cluster ingresó un número reducido de empresas locales y nacionales, proceso que fue cancelado en la tercera etapa como resultado de la intensa radicación de contratistas manufactureros globales. ${ }^{13}$

\section{La aglomeración de Guadalajara como cluster abierto}

La aglomeración industrial en las afueras de Guadalajara y los municipios aledaños debe conceptuarse como abierto o sin fronteras. Ese término es portador de la idea de que el cluster se estructura a partir de las actividades de las empresas trasnacionales y sus cadenas globales de producción, pero abre potencialmente las puertas a la iniciativa local. ${ }^{14}$ No se trata de un distrito industrial, ya que las empresas líderes son las filiales de grandes

13 El agotamiento de este medio de aprendizaje provocó desaliento entre los especialistas. Desde la perspectiva de fines de los noventa, Palacios (ibid.:334) señalaba que se había desvanecido la posibilidad de constituir un cuarto círculo de proveedores con pequeñas empresas locales innovadoras. Para Rivera Vargas y Maldonado (2004:206), la experiencia de Guadalajara corroboraba la hipótesis de que las empresas proveedoras de países en desarrollo se limitaba al estadio de imitación por duplicación.

14 Es el concepto propuesto por Green et al. (2001) para estudiar el cluster de Galway en Irlanda, especializado en productos de tecnología de la información y la comunicación. Esta expresión pretende combinar la actuación de agentes globales, como las CTN, con la existencia de capacidades locales potenciales en países en desarrollo, desarrollo medio, de reciente industrialización o, incluso, en regiones deprimidas de países desarrollados. La noción de abierto o sin fronteras se adoptó para analizar el caso de Guadalajara, porque permite combinar las condiciones de enganche o inserción, impulso local y construcción o soporte institucional. La interacción de estos elementos deja abierta la posibilidad de que exista progreso local o el cluster se estanque para convertirse en una locación subordinada para la valorización de las actividades de las CTN, a base de la sobreexplotación de los recursos humanos de la localidad.

\section{DeSarrollo}


corporaciones multinacionales y la estructura industrial es heterogénea; tampoco hay propensión innata a la innovación local, tal como está previsto en el modelo de Porter, aunque el dinamismo tecnológico local puede surgir como producto del desarrollo del cluster $^{15}$ No obstante lo anterior, existen desde un principio factores de atracción local ligados a ventajas competitivas tradicionales. Una vez que se produce el enganche externo (la atracción de empresas globales), el éxito del aprendizaje local dependerá del impulso interno (ventajas salariales, culturales y de localización) y de la sustentación institucional, sin perder de vista que la interacción entre ambos puede ser exitosa o fallida.

\section{Los efectos de la crisis y la reestructuración de la industria electrónica mundial en el cluster}

Como consecuencia de la sobreacumulación mundial en las industrias electrónicoinformáticas y del estallido de la burbuja especulativa asociada a la "nueva economía", se desencadenó la primera gran crisis mundial del SE-I. Con el epicentro en Estados Unidos, la crisis se extendió de fines de 2000 hasta comienzos de 2002, provocando una contracción del comercio mundial de 20\% en 2001 (Dabat y Ordóñez, op. cit.). La recuperación posterior iniciada en 2002 implicó profundos cambios en la industria electrónica mundial. Mientras que la industria de semiconductores sufrió una considerable caída y se desplomaba el comercio mundial de equipo de cómputo y telecomunicaciones (sectores vinculados a la desktop individual y a la telefonía fija) tuvo lugar una reorientación de la producción y el comercio internacional hacia los servidores de redes de computadoras, el software especializado y los servicios, el equipo móvil de computo y telecomunicaciones, la electrónica polifuncional de consumo y el instrumental electrónico industrial, científico y profesional (Dabat y Ordóñez, ibid.).

En el nivel espacial, se aceleró extraordinariamente la tendencia hacia la relocalización mundial de la producción del sector en países en desarrollo de bajos costos laborales y niveles medios de desarrollo educacional, no sólo de actividades relacionadas con el hardware (cuyo mapa mundial sufrió una transformación radical), sino también de nuevas actividades con grandes insumos de trabajo intelectual, como el I\&D y el diseño o el software. El aspecto más sobresaliente de la relocalización del hardware fue la conversión de China en potencia de la industria electrónica mundial (primer exportador de equipo de cómputo y de telecomunicaciones); en software, destaca el gran fortalecimiento de centros emergentes en servicios y productos encabezados por Irlanda, Israel, India (D'Costa, 2004) y, en general, la incorporación a la producción de nuevos países y la redefinición del papel cumplido por los países ya incorporados dentro de la división global del sector (Ordóñez, 2000a).

15 Pero tampoco contradice el modelo de Porter, porque se reconoce la importancia de la proximidad y la interacción localizada para constituir capacidades innovativas.

\section{DeSarrollo}


Cuadro 3

Exportaciones de México de productos electrónicos 2000-2003

(millones de dólares y porcentajes)

\begin{tabular}{lccccc}
\hline $\begin{array}{l}\text { Grupo de productos } \\
\text { clave cUCI }\end{array}$ & 2000 & 2001 & 2002 & 2003 & $\begin{array}{c}\text { Crecimiento } \\
2000-2003\end{array}$ \\
\hline $\begin{array}{l}\text { Computadoras y equipo } \\
\text { de oficina (751, 752, 759) }\end{array}$ & 11757 & 13216 & 12192 & 13370 & 13.7 \\
$\begin{array}{c}\text { Equipo de } \\
\text { telecomunicaciones (764) }\end{array}$ & 11324 & 10980 & 9284 & 7603 & -33.0 \\
$\begin{array}{l}\text { Semiconductores } \\
(776)\end{array}$ & 3064 & 2045 & 1892 & 2172 & -29.1 \\
$\begin{array}{c}\text { Electrónica de consumo } \\
(761,762,763)\end{array}$ & 7897 & 8167 & 8882 & 8214 & 4.0 \\
$\begin{array}{c}\text { Equipo e instrumental } \\
\text { médico (872 y 774) }\end{array}$ & 1527 & 1685 & 2018 & 2460 & 61.1 \\
$\begin{array}{c}\text { Instrumentos de medición } \\
\text { y control (873 y 874) }\end{array}$ & 2008 & 2372 & 2454 & 2395 & 19.3 \\
\hline \begin{tabular}{l} 
Exportaciones totales \\
\hline
\end{tabular} & 37577 & 38466 & 37721 & 36215 & -3.6 \\
\hline
\end{tabular}

Nota: se utiliza la información de la onu y la Organización Mundial de Comercio presentada en términos de la Clasificación Uniforme del Comercio Internacional (CUCI), por ser — a diferencia de la suministrada por INEGI- directamente comparable con la de las exportaciones de los restantes países del mundo en los principales sectores de la industria electrónica. Lamentablemente, esta fuente no incluye la información de 2004 por no haber sido publicada por las organizaciones mencionadas hasta el momento de las correcciones finales del presente trabajo.

Fuente: INTRACEN: http://www.intracen.org.

Estos procesos afectan sensiblemente a la industria electrónica de exportación en México y, en particular, al cluster de Guadalajara. Al desplome del mercado mundial se sumó la arrolladora competencia china en los sectores abastecidos por producción originada en México. Las empresas trasnacionales líderes radicadas en el país iniciaron una reestructuración de sus operaciones globales, la cual modificó la naturaleza de la producción regional exportable y de los insumos de trabajos y componentes requeridos. Para analizar ese proceso, comenzaremos presentando el desempeño general de las exportaciones del sector electrónico en el marco de la competencia china (véanse cuadros 3 y 4).

Según lo indicado en el Cuadro 2 y en el último renglón del Cuadro 3, el auge de las exportaciones electrónicas de México culminó en 2001 con la exportación de aproximadamente 38 mil millones de dólares ( $24 \%$ de las exportaciones totales nacionales), mientras que, de acuerdo con el Cuadro 2, las ventas dirigidas a Estados Unidos alcanzaron su nivel más elevado entre 2000 y 2001 con un valor de 31 mil millones de dólares (casi $82 \%$ del total).${ }^{16} \mathrm{~A}$ continuación vinieron dos años de ligera disminución de las exportaciones hasta alcanzar un descenso total acumulado de $8.2 \%$ entre 2001 y 2003 o de $-3.6 \%$ entre 2000 y

16 En términos totales y considerando sólo las exportaciones de productos electrónicos, la información suministrada por las oficinas estadísticas de México y Estados Unidos no difieren sustancialmente.

\section{Desarrollo}


LA REESTRUCTURACIÓN DEL CLUSTER ELECTRÓNICO DE GUADALAJARA (MÉXICO)

Cuadro 4

Exportaciones comparadas de México y China a Estados Unidos en productos electrónicos (miles de millones de dólares, porcentajes y tasas de crecimiento)

\begin{tabular}{|c|c|c|c|c|c|c|c|c|}
\hline Sectores & $\begin{array}{l}\text { Monto } \\
2004\end{array}$ & $\begin{array}{l}\text { Variación } \\
\text { porcentual } \\
\text { anual } \\
\text { promedio } \\
2000-2004\end{array}$ & $\begin{array}{c}\text { Monto } \\
2004\end{array}$ & $\begin{array}{c}\text { Variación } \\
\text { porcentual } \\
\text { anual } \\
\text { promedio } \\
2000-2004\end{array}$ & $\begin{array}{c}\% \text { de } \\
\text { impor- } \\
\text { taciones } \\
\text { de EU } \\
2004\end{array}$ & $\begin{array}{c}\text { Monto } \\
2004\end{array}$ & $\begin{array}{c}\text { Variación } \\
\text { porcentual } \\
\text { anual } \\
\text { promedio } \\
2000-2004\end{array}$ & $\begin{array}{r}\% \text { de } \\
\text { impor- } \\
\text { taciones } \\
\text { de EU } \\
2004\end{array}$ \\
\hline $\begin{array}{l}\text { Semiconductores } \\
\text { y componentes }\end{array}$ & 48.3 & -13.8 & 0.9 & -12.2 & 3.4 & 1.4 & 15.3 & 5.1 \\
\hline $\begin{array}{l}\text { Equipo de tele- } \\
\text { comunicaciones }\end{array}$ & 38.0 & -6.2 & 5.3 & -3.4 & 18.1 & 5.7 & 23.8 & 19.3 \\
\hline $\begin{array}{l}\text { Computadoras y } \\
\text { equipo de oficina }\end{array}$ & 96.0 & 0.2 & 7.8 & -4.4 & 8.1 & 36.8 & 32.7 & 38.0 \\
\hline $\begin{array}{l}\text { Electrónica } \\
\text { de consumo }\end{array}$ & 32.1 & 6.7 & 9.9 & 3.6 & 23.9 & 13.5 & 20.8 & 32.5 \\
\hline Televisores & 30.9 & 10.1 & 9.0 & 4.1 & 29.0 & 7.9 & 42.0 & 25.6 \\
\hline Radiorreceptores & 9.1 & -1.4 & 0.7 & -6.6 & 8.0 & 5.5 & 6.1 & 60.3 \\
\hline Equipo de sonido & 1.5 & 2.1 & 0.2 & 44.0 & 16.0 & 0.1 & 21.4 & 6.5 \\
\hline $\begin{array}{l}\text { Instrumental cien- } \\
\text { tífico e industrial }\end{array}$ & 17.0 & 8.8 & 2.7 & 14.5 & 11.7 & 2.1 & 5.8 & 9.0 \\
\hline Total & 217.8 & -1.5 & 26.8 & -0.5 & 12.3 & 59.4 & 32.7 & 27.3 \\
\hline
\end{tabular}

Fuente: us Census Bureau, Internacional Trade Statistics, Value of Exports, General Imports and Imports for Consummation by SITC Commodity Group, and Imports for Country, SITC, us, Mexico and China e us Infotech Statistics.

2003, como lo consigna el Cuadro 3 en la última columna. Esta caída se distribuyó muy desigualmente, con un derrumbe de las exportaciones de equipo de telecomunicaciones $(-33 \%)$ y semiconductores $(-29 \%)$ y crecimiento en computadoras (casi $14 \%$ entre 2000 y 2003) y, sobre todo, en equipo e instrumental médico (61 \%) e instrumentos de medición y control (19\%). Aunque aún no existen cifras completas para 2004, la columna 4 del Cuadro 2 indica que ha comenzado la recuperación (crecimiento de $11 \%$ en relación con 2003 de las exportaciones a Estados Unidos), especialmente en electrónica de consumo, equipo de telecomunicaciones, equipo, instrumental médico y semiconductores, con crecimiento menor en computadoras (véase us Census Bureau). Dentro del conjunto de esta

En cambio, en el nivel sectorial la información muestra fuertes discrepancias, especialmente entre el sector computadoras y equipos de oficina y el sector instrumentos de medición y control. Es muy probable que México entre en la primera categoría parte de los productos que Estados Unidos clasifica como instrumentos, lo cual hace que las cifras totales de exportación de computadoras y equipo de oficina alcancen 13370 millones de dólares en 2003, conforme las cifras suministradas por México a la ONU, y que las importaciones de ese tipo de bienes por Estados Unidos alcance sólo 7543 millones (apenas poco más de la mitad), según la información suministrada por el us Census Bureau. Lo mismo sucede en sentido contrario en el sector instrumentos, en el cual las importaciones de Estados Unidos son más elevadas que las exportaciones mundiales reportadas por México. 
evolución, destaca el reciente papel de sectores exportadores relativamente nuevos, como equipo e instrumental médico o instrumentos de medición y control, caracterizados por gran diversidad y especialización.

En lo que respecta a Guadalajara, la información de la columna 4 del Cuadro 2 muestra una evolución ligeramente diferente a la nacional. La caída comienza antes y es más aguda que la nacional (cerca de $10 \%$ en 2001), pero también comienza una recuperación más rápida. Dado su patrón de especialización, la crisis afectó más al cluster de Guadalajara que al de Tijuana, especializado en electrónica de consumo (Tvs). Pero también parece haber dado lugar a un proceso muy amplio de reestructuración en el primero, el cual explica su recuperación más rápida en comparación con las exportaciones nacionales.

La pérdida de posiciones en el mercado estadounidense en relación con China en el periodo 2000-2004 es notoria y expresa el surgimiento de esta última como potencia electrónica y el desplazamiento de nuestro país en los sectores señalados. El Cuadro 4 indica que en tanto las exportaciones de México a Estados Unidos en esas cuatro categorías disminuye $1.5 \%$, China las aumenta casi $32.7 \%$; en componentes y semiconductores el aumento a favor de esta última nación en el periodo mencionado es de $15.3 \%$, en tanto que México disminuye $13.8 \%$, lo cual le permite rebasar la participación porcentual de nuestro país; en equipo de telecomunicaciones, China aumenta sus exportaciones $23.8 \%$, lo que lo lleva a alcanzar una participación en el mercado de $20 \%$ y superar la parte de México (5.3\%); ${ }^{17}$ en computadoras y equipo, las exportaciones de China crecen más de $32 \%$, lo cual significa una participación muy superior respecto de nuestra nación (38\% contra $81 \%) .{ }^{18}$ Como ya se mencionó, los únicos sectores que continuaron creciendo firmemente en México son los que podrían considerarse nuevos (equipo e instrumental médico, así como de medición y control), en los cuales su participación es superior a la de China (11.7\% contra 9). ${ }^{19}$ Esos productos se suman a otros, como los de electrónica automotriz, aeroespacial, telecomunicaciones inalámbricas o de base óptica, nuevos dispositivos multifuncionales de la electrónica de consumo y electrónica industrial que ya habían aparecido en la producción asentada en México. ${ }^{20}$

17 En 2000 las participaciones de México y China eran de $20.3 \%$ y $10.6 \%$ respectivamente; recabado por Dabat y Ordóñez de acuerdo con información del us Census Bureau, International Trade Statistics, Mexico and China.

18 La misma relación en 2000 era de $12.1 \%$ contra 9.7\% (US Census Bureau, International Trade Statistics, Mexico and China).

19 El subsector de instrumentos reviste gran importancia no sólo debido a que el monto de sus exportaciones supera al de los componentes y semiconductores, sino principalmente a que el subsector puede convertirse en uno de los más dinámicos durante el periodo de recuperación, proceso en el cual, por tanto, México estaría bien posicionado, pues la penetración tecnológica de la electrónica en los instrumentos es de las más tardías, por lo que se trata de un subsector naciente con alto potencial de crecimiento.

$20 \mathrm{Al}$ momento no disponemos de suficiente información para saber en qué localidades se asientan los productos genéricamente designados instrumentos.

\section{DeSarrollo}


La información agregada del Cuadro 4 sugiere que el desplazamiento a China ${ }^{21}$ no fue indiscriminado, sino que siguió un modelo con dos variantes, lo cual fue confirmado en las entrevistas del trabajo de campo: $a$ ) en el caso de los productos que se elaboran en pequeñas series y contienen una alta variedad de componentes, las OEM tendieron a mantenerlos en México (los servidores, sistemas de almacenamiento y equipo para redes de telecomunicaciones). Al contrario, en lo que respecta a los productos que se manufacturan en grandes series que requieren de una variedad reducida de componentes (equipo de cómputo, equipo de telecomunicaciones excepto teléfonos celulares y partes y componentes) la producción se deslizó a China. ${ }^{22}$ De lo anterior resulta que el cluster haya encontrado una salida a la crisis en el ascenso en las cadenas de valor globales, orientado a procesos de manufactura más intensivos en conocimiento y de mayor valor agregado; $b$ ) demanda de servicios intensivos en conocimiento que requieren trabajo técnico de alto nivel y de ingeniería a las cuales se hizo referencia anteriormente. Es en ese rubro, en el cual juega un papel relevante un pequeño grupo de empresas locales, como veremos más adelante.

El desplazamiento a China puede explicarse con apoyo del concepto de costo relacional, como se verá a continuación.

Debido a que existe una relación directamente proporcional entre los costos relacionales — es decir, los de la relación operativa entre la empresa OEM y su $\mathrm{CM}^{23}$ y la complejidad tecnológica de los productos y, en cambio, una relación inversamente proporcional entre el volumen de las series de productos y los costos de transporte, las ventajas competitivas de México en relación con la producción no relocalizada (elaborada en Estados Unidos) y la de relocalización lejana (en Asia y, específicamente, China) radica en productos que se hacen en medianas series y contienen alta variedad de componentes electrónicos, debido a que el mayor costo unitario de producción en relación con la relocalización lejana es compensado por menores costos unitarios relacionales y menores costos unitarios de transporte, como se muestra en el Cuadro 5.

21 El desplazamiento a China fue producto de una estrategia global, pero se vio agudizada por el incremento de los costos laborales en México y la apreciación cambiaria. Con base 1990, el índice del costo laboral unitario pasó de 56.3 en 1999 a 70.4 en 2001 (Dabat y Ordóñez, op. cit.).

22 En este caso la reducción de costos se deriva principalmente del acceso a componentes asiáticos de bajo costo, los cuales constituyen aproximadamente $79 \%$ de los costos de producción totales de un circuito impreso y, marginalmente, de los costos laborales que representan 10\% (Electronic Business, 1 de marzo de 2005).

23 El costo relacional incluye todos los costos operativos derivados de que no realicen las empresas OEM sus propios procesos de manufactura de sus productos, sino que los subcontraten con manufactureros.

\section{DeSarrollo}


Cuadro 5

Ventajas competitivas de Estados Unidos, México y China según la complejidad tecnológica del producto y el volumen de producción para las empresas oEM

\begin{tabular}{|c|c|c|c|}
\hline & Estados Unidos & México & China \\
\hline \multicolumn{4}{|c|}{$\begin{array}{l}\text { Producto A: pequeña serie/alta variedad de componentes, } \\
\text { ECI* y fabricación de la caja (gran gabinete para la industria } \\
\text { médica). Volumen anual medido en } 1000\end{array}$} \\
\hline Precio venta/fábrica FOB & \$1984 & $\$ 1945$ & $\$ 1938$ \\
\hline Costo de transporte & 0 & 17 & 26 \\
\hline Costo relacional** & 30 & 73 & 110 \\
\hline Costo empresa OEM & 2014 & 2035 & 2074 \\
\hline \multicolumn{4}{|c|}{$\begin{array}{l}\text { Producto } B \text { : mediana serie/alta variedad de componentes, } \\
\text { ECI* para producto de telecomunicaciones. Volumen anual } \\
\text { medido en } 10000\end{array}$} \\
\hline Precio venta/fábrica FOB & 1307 & 1284 & 1272 \\
\hline Costo de transporte & 1 & 3 & 5 \\
\hline Costo relacional $* *$ & 9 & 23 & 39 \\
\hline Costo empresa OEM & 1317 & 1310 & 1316 \\
\hline \multicolumn{4}{|c|}{$\begin{array}{l}\text { Producto } C \text { : gran serie/reducida variedad de componentes } \\
\text { ECI* y fabricación de caja la para producto de electrónica } \\
\text { de consumo. Volumen anual medido en } 100000\end{array}$} \\
\hline Precio venta/fábrica FOB & 120.77 & 100.40 & 92.40 \\
\hline Costo de transporte & 0.45 & 0.88 & 1.10 \\
\hline Costo relacional** & 0.56 & 1.57 & 2.36 \\
\hline Costo empresa OEM & 121.78 & 102.85 & 95.86 \\
\hline
\end{tabular}

* Ensamble de circuito impreso.

** Costo operativo de la relación de la empresa OEM-ODM con la empresa CM.

Fuente: Electronic Business, 1 de marzo de 2005.

En síntesis, la reestructuración del cluster se caracteriza por las siguientes tendencias:

a) Ascenso industrial hacia el diseño, con las modalidades de creación de centros de diseño y prueba de producto por parte de las empresas oEM globales como Intel, ST Semiconductors o Global Vantage; ${ }^{24}$

b) ascenso industrial hacia la manufactura de procesos más intensivos en conocimiento y de mayor valor agregado, los cuales resultan en productos tecnológicamente más complejos integrados en sistemas, como los servidores, los sistemas de almacenamiento de información y la producción de equipo para redes de telecomunicación (en relación

24 Intel funda un centro de diseño de semiconductores para telecomunicaciones en 2000 y ST Semiconductors planea hacerlo en 2005. Las empresas locales que se integran en el proceso lo hacen en nichos específicos, en los que llevan a cabo diseño propio o actúan como contratistas en el diseño de semiconductores, circuitos impresos, hardware y software (CADELEC, 2004 y entrevistas propias realizadas en noviembre de 2004).

\section{DeSarrollo}


con el ensamble previo de computadoras o de teléfonos celulares, por ejemplo), ${ }^{25}$ con una modalidad de producción de pequeñas o medianas series; ${ }^{26}$

c) reorientación hacia sectores y subsectores emergentes o de alto dinamismo como instrumentos, electrónica automotriz, aeroespacial, telecomunicaciones inalámbricas o de base óptica, nuevos dispositivos multifuncionales de la electrónica de consumo e industrial.

d) fortalecimiento de una nueva modalidad de empresa local oEM, la cual lleva servicios avanzados para empresas OEM-ODM globales, como diseño de software tipo soluciones, ${ }^{27}$ prueba de producto y diseño de circuitos integrados y hardware avanzado; ASCI sobresale en software tipo soluciones. Otras empresas OEM locales han desarrollado las siguientes capacidades: 1) diseño de software y hardware, como Resser, Quest y ATR; 2) diseño y manufactura de equipos de telecomunicaciones para redes de acceso como Mixbaal; 3) diseño de equipo de prueba como INSOL; y 4) diseño de circuitos impresos y electrónico como Seriie y Gollet (CADELEC, 2004). Estas empresas han efectuado lo que llamaremos segunda modalidad de aprendizaje, para distinguirlo del realizado en partes y componentes en la segunda etapa del desarrollo del cluster, cuestión que se abordará con profundidad más adelante.

\section{La nueva modalidad del aprendizaje y su base institucional: el papel del Centro de Tecnología de Semiconductores (стS) y de la Cadena de la Industria Electrónica (CADELEC)}

Varias de las empresas locales a las cuales nos referimos en el párrafo anterior surgieron en el auge de los noventa, pero se consolidaron en el contexto de la reestructuración de 20012003 y en la expansión subsiguiente. ${ }^{28}$ ASCI, por ejemplo, fue fundada en 1993 y hasta 2001 tuvo 35 empleados para pasar a 100 en $2004 .{ }^{29}$ El contexto que posibilitó su crecimiento acelerado y de las restantes empresas del mismo tipo persiste y existen nuevos proyectos en gestación encabezados por ingenieros que adquirieron experiencia en empre-

25 Por ahora, los celulares son una excepción porque la regla de origen establecida por el TLCAN para los circuitos impresos de teléfonos celulares se renegoció en 2004, con el resultado de que se le impuso a los productos chinos un arancel de 18\%. En consecuencia, Nokia regresó su producción a México (declaraciones del director general de Jabil Circuits, Ernesto Sánchez Proal).

26 En la terminología empresarial, se trata de producción de bajos volúmenes con alta mezcla, en relación con las grandes series de productos poco variados, en las cuales países de más bajo costo laboral internacional y gran capacidad instalada, como China, tienen ventajas con las que México no puede competir.

27 El software tipo soluciones es el que capacita a la computadora y a los artefactos que funcionan con base en microprocesadores para desarrollar ciertas funciones requeridas por el usuario final (Mowery, 1999:133).

28 Punto en el cual coincidieron los directivos de las oEM locales entrevistados.

29 Entrevista con Miguel A. Casillas, responsable de desarrollo de negocios de AscI.

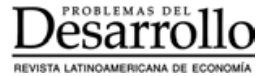


sas trasnacionales, pero principalmente en conexión con el puntal tecnológico de la región: CTS. $^{30}$

Ese papel le confiere al CTS un rango institucional sin precedentes en México, que curiosamente ha sido poco discutido académicamente. Varias de las nuevas empresas fueron fundadas y están dirigidas por ingenieros-empresarios formados en el cTs. En un análisis se argumenta que el retiro de IBM obligó al CTS a desligarse de las actividades locales y se orientó al mercado externo (Dussel, op. cit::63). Lo anterior es cierto, pero también el CTs ha sido el crisol del cual salió el talento en ingeniería y las conexiones con empresas globales, fundamentos en el segundo medio de aprendizaje. De acuerdo con Francisco de la Torre, del CINVESTAV, la búsqueda internacional de clientes realizada por el laboratorio una vez que se retiró IBM, en lugar de dispersar a los ingenieros mexicanos, aumentó su arraigo local y el interés de firmas externas en los recursos del cluster.

Por medio de CTS se han canalizado numerosos proyectos externos hacia el cluster y la red de relaciones profesionales gestada en su interior ha permitido asegurar el soporte técnico y profesional de las nuevas empresas (start-ups). ${ }^{31}$ Mixbaal es el ejemplo más sobresaliente (véase Cuadro 6), pero también lo es DTcom, especializada en diseño y prueba de producto. Esta última fue creada por un ingeniero que adquirió experiencia en CTS, Jesús Palomino Echartea, apoyándose en las conexiones y capacidad tecnológica del laboratorio. La reputación del personal llamó la atención de INTEL, quien compró a DTCom para convertirlo en centro regional de diseño, confiriéndole sustancial autonomía a Palomino y su equipo. Aunque Sergio Fernández, fundador de ASCI, no tuvo una etapa formativa en CTS, se benefició de la relación entre ésta y HP, el cliente más importante del laboratorio después del retiro de IBM. ${ }^{32}$ Dentro de la literatura de coordinación cognoscitiva e institucional de los clusters, podría definirse al cTS como eje de un incipiente sistema regional de innovación, ya que ha actuado como enlace entre empresas globales, locales, gobierno local, personal especializado, asociaciones empresariales e instituciones educativas. ${ }^{33}$

CADELEC es la otra institución que expresa el nuevo activismo colectivo en el cluster y contribuyó a la reestructuración de la cadena de proveedores que se produjo en respuesta a la crisis. Fue fundada en 1997 como asociación civil, por iniciativa de las principales oEMs y CMS de la localidad (IBM, HP, Intel, Jabil Circuit y Solectrón, que son sus fundadores y patrocinadores), contando con el apoyo de la CANIETI y del gobierno del estado. Su función consiste en desarrollar e integrar empresas proveedoras, sin discriminar si son locales, nacionales o extranjeras. Para llevar a cabo esa función posee una metodología

30 Entrevista con Francisco de la Torre, CINVESTAV CTS.

31 Ibidem.

32 Ibid.

33 El valor agregado por estas empresas es sustancialmente superior a las del primer aprendizaje, como se deduce de la participación de ingenieros en la fuerza laboral, pero no hemos efectuado estimaciones comparativas.

\section{DeSarrollo}


para que las empresas postulantes obtengan certificación internacional, crédito, asistencia técnica y capacitación especializada. CADELEC evalúa las propuestas de proveedores potenciales, partiendo de las necesidades de sus patrocinadores, los cuales participan en las reuniones de arranque, seguimiento y cierre de los proyectos de evaluación. El costo mensual por consultoría especializada de diez meses de duración es de 2000 dólares, aproximadamente, de los cuales hay un reembolso (con apoyo estatal y federal) de 50\% para pequeñas y medianas empresas. ${ }^{34}$

La demanda global de servicios avanzados (desarrollo de software, diseño y prueba de sistemas, entre otros) ha crecido explosivamente en los últimos diez años como producto de las necesidades de interconexión, integración y comunicación entre sistemas que acompaña el predominio del software sobre el hardware (The Economist, Survey, 13 de noviembre de 2004). La constitución de nichos para la producción de servicios de software, así como sus productos, ha tendido a reubicarse en economías emergentes, entre las cuales sobresalen India, Filipinas, Brasil, Irlanda e Israel. Si consideramos que esos servicios y productos se pueden clasificar por el valor añadido por las empresas locales en niveles alto, medio y bajo, México se ubica en la categoría media (desarrollo de soluciones, software para integración de sistemas y para prueba de producto), en contraposición con India, donde predominan los de bajo valor agregado (gestión de legados, mantenimiento, migración e information technology enabled services (ITES), aunque con tendencia a desplazarse hacia el escaño superior. ${ }^{35}$ Las empresas locales en Irlanda, por su parte, se especializan en la producción de software empaquetado para empresas OEM-ODM (D'Costa, 2004 y Sridharan, 1996).

Como se advierte en la información del Cuadro 6, el proceso de aprendizaje de estas nuevas empresas difiere sustancialmente del que siguieron las empresas locales tradicionales, porque poseen activos de conocimiento creados a partir de la alta formación y calificación de la fuerza laboral (proporción dominante de ingenieros en la fuerza laboral). ${ }^{36}$

Considerando que es posible trasladar las capacidades tecnológicas acumuladas de un segmento a otro del mercado de software de aplicación al software de Internet o al desarrollo de productos (Casper y Glimstedt, 2001), el potencial de crecimiento de las empresas locales es considerable. El reto consiste en integrase a las redes de subcontratación y aprovechar las oportunidades de subcontratación que ofrecen las empresas OEM.

34 Información en CD de presentación, complementada con entrevista a Jacobo González, director general de CADELEC.

35 En cuanto a México, no debe olvidarse que, pese a este avance local, el país se encuentra más atrasado en la producción y servicios de software que Brasil y Argentina, por ejemplo (Mochi, 2004).

36 De los 100 empleados que tuvo ASCI en 2004, cuatro tienen grado de doctor (electrónica, sistemas y óptica), veinte son maestros en ciencias computacionales, diez son técnicos y el resto - 66-, ingenieros (entrevista con Miguel A. Casillas, ASCI).

\section{DeSarrollo}


Cuadro 6

Actividades de empresas proveedoras de servicios avanzados (mediados de la década de 2000)

\begin{tabular}{|c|c|c|c|c|c|}
\hline & $\begin{array}{c}\text { Capacidades } \\
\text { en diseño }\end{array}$ & $\begin{array}{c}\text { Flujos de } \\
\text { de innovaciones } \\
\text { incrementales } \\
\text { patentables }\end{array}$ & $\begin{array}{c}\text { Actividades de } \\
\text { investigación } \\
\text { y desarrollo }\end{array}$ & $\begin{array}{c}\text { Proyectos } \\
\text { regulares con } \\
\text { clientes globales }\end{array}$ & $\begin{array}{l}\text { Alta proporción } \\
\text { de ingenieros en } \\
\text { la fuerza laboral }\end{array}$ \\
\hline $\mathrm{ASCI}^{*}$ & Alta & $\mathrm{x}$ & $\mathrm{x}$ & $\mathrm{x}$ & $\mathrm{x}$ \\
\hline MIXBAAL $* *$ & Alta & $\mathrm{x}$ & $\mathrm{x}$ & $\mathrm{x}$ & $\mathrm{x}$ \\
\hline
\end{tabular}

* ASCI fue fundada por Sergio Fernández, doctorado en ciencias computacionales en Estados Unidos; la empresa se ha ido constituyendo como laboratorio de desarrollo y prueba de software, prueba equipo de cómputo y periféricos, interconexión de equipos, tecnología de reconocimiento de imagen, entre otros. Su principal cliente es HP, para quien desarrolla diversos tipos de software, principalmente de aplicación, para manejo de hojas de impresora, proceso sobre el cual tiene un prestigio continental. Tiene ocho patentes en proceso.

** MIXBAAL fue creada en 1994 por un grupo de ingenieros que se desarrolló profesionalmente en CINVESTAVCTS, al frente de quienes está J.M. Garduño, cofundador del laboratorio y su director hasta 1997. Se especializa en diseño y manufactura de equipos de telecomunicaciones para redes de acceso. Su principal cliente es TELMEX, pero es dueña de una firma estadounidesne que utiliza para obtener contratos internacionales.

Fuente: entrevistas realizadas por el equipo coordinado por Dabat, Rivera y Ordóñez en Guadalajara; los entrevistados fueron Miguel A. Casillas para ASCI, y J.M. Garduño para MIXBAAL.

Paradójicamente, la consolidación del dominio chino en cuanto a los productos electrónicos de alto volumen tuvo el efecto de consolidar la posición inicial de las nuevas empresas mexicanas. Este nuevo medio de aprendizaje no podrá afianzarse si no sucede con el incipiente sistema regional de innovación centrado en cTs. Otra paradoja, que expresa la falta de lo que los agentes locales del cluster llaman "falta de una estrategia nacional para la industria" es la ausencia de un proyecto de crecimiento de ese laboratorio. ${ }^{37} \mathrm{Al}$ no existir este último, el talento empresarial, científico y de ingeniería quedará corto ante la demanda de servicios globales que se orienta en una medida cada vez mayor hacia los centros emergentes. Trataremos esa cuestión en un marco más amplio.

\section{Conclusión}

El cluster electrónico de Guadalajara transita por tres etapas de desarrollo que van desde finales de los años sesenta del siglo xx hasta el auge exportador de 2000. Una tercera etapa empieza a constituirse a partir de la crisis y la reestructuración del cluster. Durante la segunda etapa (desde mediados de los ochenta) tiene lugar una primera modalidad de aprendizaje tecnológico de las empresas locales, basado en la formación de sistemas básicos de conocimiento, enfocados en la incorporación desde abajo en las cadenas de valor

37 CTS que ha tenido un récord impresionante en el diseño de circuitos integrados y el desarrollo de tarjetas, para clientes globales, no ha rebasado, desde su creación, el tamaño de treinta ingenieros. Considerando que CTS ha cumplido en una escala minúscula un papel equivalente a los laborato rios creados por el Instituto Avanzado de Ciencias y Tecnología de Corea del Sur (Kim, 1997), su crecimiento debiera haberse planteado como parte de la reestructuración del cluster. Ninguno de los entrevistados dio respuesta satisfactoria a esta observación.

\section{Desarrollo}


como proveedores de partes y componentes. Esta modalidad de aprendizaje queda prácticamente cancelada en la tercera etapa de desarrollo del cluster, que da sustento al auge exportador durante la segunda mitad de los años noventa, fundada en la radicación de los contratistas manufactureros globales de las empresas oEm existentes en el cluster y de sus proveedores internacionales de partes y componentes.

La crisis y reestructuración de la industria electrónica mundial (que aún continúa) tiene el efecto de precipitar una reestructuración del cluster de Guadalajara, consistente en: a) un ascenso industrial hacia el diseño y la manufactura de procesos más intensivos en conocimiento, los cuales dan lugar a productos tecnológicamente más complejos integrados en sistemas, con una modalidad de producción de pequeñas o medianas series, lo que diferencia al país de China, es decir, de uno de sus principales competidores, en el cual radica la elaboración de productos de menor valor agregado, fabricados en grandes series y que requieren de una reducida variedad de componentes; $b$ ) reorientación hacia sectores y subsectores emergentes o de alto dinamismo como instrumentos, electrónica automotriz, aeroespacial, telecomunicaciones inalámbricas o de base óptica, nuevos dispositivos multifuncionales de la electrónica de consumo y electrónica industrial; y $c$ ) fortalecimiento de una nueva modalidad de empresa local oDM, que se inserta en el proceso de relocalización-subcontratación internacionales de las actividades de I\&D que demandan mayores insumos de conocimiento.

La empresa ODM local emergente lleva a cabo lo que se ha denominado una segunda modalidad de aprendizaje tecnológico, el cual se caracteriza por una inserción en la cadena de valor desde arriba. Esa inserción superior tiene un sustento institucional representado por CTS y CADELEC, así como de la relación y asistencia técnica con las empresas oEM globales. Éstas se apoyan en recursos institucionales en educación, capacitación e investigación y desarrollo centralizados en cierta medida en el cTs, las cuales, ciertamente, estaban latentes con mucha anterioridad, pero que detonan en el contexto de la crisis y la reestructuración subsiguiente.

Recordemos que el cTs fue creado por una iniciativa estatal en coordinación con la entonces empresa líder, IBM; después, el apoyo público se redujo a su mínima expresión, pero el Centro pudo actuar como eje de acumulación de capacidades tecnológicas gracias a que interiorizó las externalidades positivas creadas por la aglomeración.

El ascenso industrial y el nuevo aprendizaje tecnológico en el cluster de Guadalajara, aún de carácter embrionario, es de suma importancia y pone de manifiesto la urgencia de una estrategia nacional de desarrollo de la productividad y la competitividad en sectores de frontera, basadas en el conocimiento, sin las cuales el proceso que está en marcha en Guadalajara podría sustentarse a largo plazo ni extenderse territorialmente. Se trata, por tanto, de un tema que demanda gran atención de los especialistas, pero requiere también ser enfocado mediante nuevos instrumentos de política industrial que se inscriban en el marco de los llamados sistemas regionales de innovación.

\section{DeSarrollo}




\section{Bibliografía}

Balassa, Bela, "La política de comercio exterior en México", en Comercio Exterior, marzo de 1983.

Bell, M. y M. Albu, "Knowledge Systems and Technological Dynamism in Industrial Clusters in Developing Countries", en World Development, vol. 27, núm. 9, septiembre de 1999, pp. 17151734.

CADELEC, Presentación de la cadena de la industria electrónica en Guadalajara, 2004.

Casper, S. y H. Glimstedt, "Economic Organization and Innovation System, and the Internet", en Oxford Review of Economic Policy, vol. 17, núm. 2, 2001.

Cooke, Philip, "Regional innovation System, Clusters and the Knowledge Economy", en Industrial and Corporate Change, vol. 10, núm. 4, 2001, pp. 945-974.

Dabat, Alejandro, "Economía informática y nueva industria electrónica de exportación en México", en Problemas del Desarrollo, vol. 53, núm. 137, abril-junio de 2004.

y Rivera R., Miguel A, "Nuevo ciclo industrial mundial e inserción de países en desarrollo", en A. Dabat, M. Rivera y J. Wilkie (coordinadores), Globalización y cambio tecnológico. México en el nuevo ciclo industrial mundial, México, UdG, UNAM, UCLA, PROFMEX y Juan Pablos, 2004.

- y S. Ordóñez, "Revolución informática, nuevo ciclo industrial y división internacional del trabajo", Cuadernos de Investigación, CRIMUNAM, en prensa.

D’Costa, Anthony, "The Indian Software Industry in the Global Division of Labour', en A. D'Costa, y E. Sridharan (editores), India in the Global Software Industry. Integration, Firms Strategies and Development, Londres, Palgrave Macmillan, 2004.

Dussel, E., "Ser maquila o no ser maquila ¿es ésa la pregunta?", en Comercio Exterior, vol. 91, núm. 4, 2003, pp. 329-336.

, Polarizing Mexico. The Impact of Liberalization Strategy, Boulder, Lynne Rienner, 2000.

_- La subcontratación como proceso de aprendizaje: el caso de la electrónica en Jalisco (Mexico) en la década del noventa, Santiago, CEPAL, 1998.

Ernst, Dieter, "Redes globales de producción, difusión de conocimiento y formación de capacidades locales. Un marco conceptual”, en E. Dussel, J. Palacios y G. Woo (coordinadores), La industria electrónica en Mexico, México, Universidad de Guadalajara, 2003.
Fajnzylber, Fernando, La industrialización trunca de América Latina, México, Nueva Imagen, 1983.

Gereffi, Gary, "La transformación de la industria de la confección en América del Norte", en A. Dabat, M. Rivera y J. Wilkie (coordinadores), Globalización y cambio tecnológico. Mexico en el nuevo ciclo industrial mundial, UDG, UNAM, UCLA, PROFMEX y Juan Pablos, 2004.

, "Global Production System and Third World Development", en Barbara Stallings (editora), Global Change, Regional Response, Cambridge, Cambridge University Press, 1995.

Green, Roy, I. Duggan, M. Giblin, M. Moroney y L. Smyth, "The Boundaryless Cluster: Information and Communication Technology in Ireland", en OECD, Innovative Clusters, Drivers of National Innovation System, París, OECD, 2001.

Hobday, Michael, Innovation in East Asia. The Challenge to Japan, Aldershot, Edward Elgar.

INEGI, Sistema de cuentas nacionales, México, 1995.

Kaplisnky, R., Globalisation, Industrialisation and the Pursuit of the NTH Rent. IDS, Discussion Paper, núm. 365, Sussex, Institute of Development Studies, 1998.

_ Republic: Transforming Manufactures into Commodities", en World Development, vol. 21, núm. 11, 1993.

Kim, Linsu, Imitation to innovation. The Dynamics of Korea's Technological Learning, Boston, Harvard Business Scholl Press, 1997.

Koido, Akihiro, "La industria de televisores a color en la frontera de México con Estados Unidos: potencial y límites del desarrollo local", en Comercio Exterior, vol. 53, núm. 4, 2003, pp. 356-372.

Mochi, Prudencio, "La industria del software en México", en Problemas del Desarrollo, vol. 53, núm. 137, abril-junio de 2004.

Mowery, David, "The computer software industry", en D. Mowery y R. Nelson (editores), Sources of industrial leadership. Studies fo seven industries, Cambridge, Cambridge University Press, 1999.

Ordóñez, Sergio, "La nueva división interindustrial del trabajo y empresas electrónicas en México", en A. Dabat, M. Rivera y J. Wilkie (coordinadores), Globalización y cambio tecnológico. México en el nuevo ciclo industrial mundial, México, UDG, UNAM, UClA, PROFMEX y Juan Pablos, 2004.

, "La nueva fase del desarrollo y el capitalismo del conocimiento: elementos teóricos", en Comercio Exterior, vol. 24, núm. 1, enero, 2004a. 
Palacios Lara, Juan, "La industria electrónica en Jalisco: ¿de aglomeración desarticulada a complejo industrial integrado?", en Enrique Dussel, J. Palacios y G. Woo (coordinadores), La industria electrónica en México: Problemática, perspectivas y propuestas, México, Universidad de Guadalajara, 2003.

-, Production Networks and Industrial Clustering. Electronic Manufacturing in Guadalajara, Universidad de Guadalajara, 2001.

Peres Núñez, Wilson, Foreign Direct Investment and Industrial Development in Mexico, París, OECD, 1990.

Pérez, Carlota, Cambio tecnológico y oportunidades de desarrollo como blanco móvil”, en Revista de la CEPAL, núm. 75, diciembre de 2001, pp. 115-136.

Reinhardt, Nola y Wilson Peres, "Latin America's New Economic Model: Micro Responses and Economic Restructuring", en World Development, vol. 28, núm. 9, 2000, pp. 1543-1565.

Rivera Ríos, Miguel A., "La inserción de México en la economía global", en J. Basave et al. (coordinadores), Globalización y alternativas incluyentes para el siglo XXI, México, UNAM, UAMI, Porrúa, 2002.

, México en la economía global. Tecnología, espacio einstituciones, México, UNAM, UCLA, Jus, 2000.

Rivera Vargas, María y J. Maldonado, “Aprendizaje tecnológico en los proveedores de la industria electrónica, Guadalajara, México", en Comercio Exterior, vol. 54, núm. 3, 2004, pp. 196-206.

Secretaría de Economía, Programa para la competitividad de la industria electrónica y de alta tecnología, México, 2003.

Sridharan, E., The Political Economy of Industrial Promotion: Brazilian, and Korea Electronics in Comparative Perspective, 1969-1994, Westport, Praeger, 1996.

Sturgen, Timothy, "Modular Production Networks: a New American Model of Industrial Organization", en Industrial and Corporate Change, vol. 11, núm. 3, 2002.

Warman, José, "La competitividad de la industria electrónica: situación y perspectivas", en F. Clavijo y J. Casar, La industria mexicana en el mercado mundial. Elementos para una política industrial, México, FCE, 1994.

\section{DeSarrollo}

